EXTENDED REPORT

\title{
Radiological hand involvement in systemic sclerosis
}

\author{
J Avouac, H Guerini, J Wipff, N Assous, A Chevrot, A Kahan, Y Allanore
}

Ann Rheum Dis 2006;65:1088-1092. doi: 10.1136/ard.2005.044602

\begin{abstract}
See end of article for authors' affiliations .....................

Correspondence to: Dr Allanore Yannick Hôpital Cochin, Service de rhumatologie A, 27 rue du faubourg Saint Jacques, 75014 Paris, France yannick.allanore@cch.aphop-paris.fr
\end{abstract}

Accepted

30 December 2005

Published Online First

13 January 2006

Background: The osteoarticular and soft tissue structures of the hand may be involved in systemic sclerosis (SSc), causing functional disability.

Objective: To assess radiological hand features in a cross sectional study of SSc patients and in controls. Methods: Hand radiology was done systematically in patients with SSc seen over a two year period and in unselected controls with rheumatoid arthritis or digital trauma. Two independent investigators blind to the diagnosis carried out the radiological assessment.

Results: 120 consecutive SSc patients (median (range) age, 56.5 (20 to 90) years; disease duration, 6 (0 to 42 ) years) and 42 controls (22 with rheumatoid arthritis and 20 with digital trauma) were studied. Radiological abnormalities in SSc patients included erosion (21\%), joint space narrowing (28\%), arthritis (defined by concomitant erosion and joint space narrowing) (18\%), radiological demineralisation (23\%), acro-osteolysis $(22 \%)$, flexion contracture $(27 \%)$, and calcinosis $(23 \%)$. In univariate and multivariate analysis, the resorption of distal phalanges was significantly associated with digital ulcers, extra-articular calcification, and pulmonary arterial hypertension; flexion contracture was associated with the diffuse cutaneous form and high HAQ (Health Assessment Questionnaire) disability score. Calcinosis was most often seen in patients with digital ulcers, but was similarly observed in patients with the diffuse or limited cutaneous subtypes.

Conclusions: Flexion contracture was associated with disability and occurred in patients with the diffuse cutaneous subtype of SSc, consistent with the tendency towards fibrosis and functional impairment of this subtype. Calcinosis and acro-osteolysis were both associated with vascular complications, highlighting a potential role of vascular injury in such lesions.

S ystemic sclerosis (SSc) is a connective tissue disease characterised by vascular, immune, and fibrotic changes in the skin and some internal organs. Joint involvement has been reported to occur in up to $46 \%^{1}$ or $97 \%^{2}$ of SSc patients. Many distinct radiographic abnormalities have been recognised, ${ }^{1-8}$ but the prevalence and distribution of joint involvement have not been accurately defined. ${ }^{3}$ Bone and soft tissues may also be targeted; calcinosis and acro-osteolysis are thought to be the main characteristic findings in $\mathrm{SSc}^{13}$ 9-14 but little is known about their distribution or their association with disease phenotype. Hand involvement in SSc is disabling ${ }^{15}$ as recently demonstrated by functional analysis. ${ }^{16-}$ ${ }^{20}$ Increasing our understanding of osteoarticular involvement may make it possible to improve therapeutic approaches.

Our aim was first, to determine the frequency of radiological articular, bony, and soft tissue hand involvement in a cross sectional study with prospective data collection of SSc patients, with the inclusion of controls in order to limit the bias of radiological analysis; and second, to identify disease-phenotype associations.

\section{METHODS}

All consecutive SSc patients referred to the "Rheumatology $\mathrm{A}^{\prime \prime}$ department over a period of two years were included. We carried out a global evaluation of these patients, based on collection of the following data: age, sex, dominant hand, cutaneous SSc subtype as defined by LeRoy et al, ${ }^{21}$ disease duration (date of first non-Raynaud symptom), duration of Raynaud's phenomenon, digital ulceration, and prostacyclin use. The French version of the Health Assessment Questionnaire (HAQ) was used to evaluate functional disability. Systemic assessment was carried out as recommended. ${ }^{22}$ Pulmonary fibrosis was assessed by computed tomography (CT) and respiratory function tests (forced vital capacity (FVC) and carbon monoxide diffusion capacity divided by alveolar volume (DLCO/VA)). Pulmonary arterial hypertension (PAH) was defined as pulmonary arterial pressure higher than $40 \mathrm{~mm} \mathrm{Hg}$ at rest on Doppler echocardiography. ${ }^{23}$ The following biological tests were carried out: routine blood tests, tests for antinuclear and anti-centromere antibodies (immunofluorescence on Hep2 cells), anti-topoisomerase I and anti-RNP antibodies (counter immunoelectrophoresis), rheumatoid factor (RF; enzyme linked immunosorbent assay (ELISA)), anti-keratin antibodies (AKA; indirect immunofluorescence), and second generation anti-cyclic citrullinated peptide antibody (anti-CCP2; ELISA).

We limited inclusion bias by including all unselected patients admitted to hospital for rheumatoid arthritis fulfilling the American College of Rheumatology (ACR) criteria, and successive subjects consulting for digital trauma. All patients gave informed consent for all procedures.

Standard anteroposterior views of the hands and wrists were obtained for all patients. Three radiological patterns of abnormalities of joints (erosion: interruption of the cortical surface; space narrowing: focal or diffuse joint narrowing), bone (radiological demineralisation: juxta-articular or generalised osteoporosis; bone resorption), and soft tissue

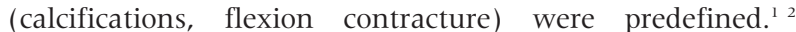
Proximal interphalangeal (PIP) and distal interphalangeal (DIP) joints were not taken into account for the evaluation of joint space narrowing in cases of digital contracture. Radiographs were evaluated by two independent investigators blind to the diagnosis. Any discrepancies were resolved by a consensus reading with a third independent observer.

Abbreviations: ACR, American College of Rheumatology; AKA, antikeratin antibodies; DIP, distal interphalangeal joint; $H A Q$, Health Assessment Questionnaire; PAH, pulmonary arterial hypertension; RF, rheumatoid factor; SSc, systemic sclerosis 
Table 1 Characteristics of the patients with systemic sclerosis

\begin{tabular}{ll}
\hline Variable & $\begin{array}{l}\text { SSc patients } \\
\text { (n= } 120)\end{array}$ \\
\hline $\begin{array}{l}\text { Duration of Raynaud's phenomenon (years) } \\
\text { (median (range)) }\end{array}$ & $10(0$ to 42$)$ \\
Digital ulcers/prostacyclin use & $37(31 \%) / 20(17 \%)$ \\
HAQ (mean (SD)/n patients $>1.5(\%)$ & $1.1(0.8) / 29(24 \%)$ \\
Pulmonary arterial hypertension & $17(14 \%)$ \\
Pulmonary fibrosis on CT & $58(48 \%)$ \\
C reactive protein $>10$ mg/l & $21(17.5 \%)$ \\
Positive anti-nuclear antibodies (>1/160) & $87(74 \%)$ \\
Positive anti-topoisomerase I antibodies & $36(30 \%)$ \\
Positive anti-centromere antibodies & $21(18 \%)$ \\
Positive rheumatoid factor & $32(27 \%)$ \\
Positive AKA/anti-CCP2 antibodies & $0(0 \%) / 2(1.7 \%)$ \\
Decreased FVC (<75\% of normal value) & $29(25 \%)$ \\
Decreased DLCO/VA ( $<75 \%$ normal value) & $44(37 \%)$ \\
Low dose prednisone & $45(37 \%)$ \\
Methotrexate & $17(14 \%)$ \\
\hline
\end{tabular}

Values are $\mathrm{n}(\%)$ unless stated otherwise.

Anti-CCP, anti-cyclic citrullinated peptide antibody; AKA, anti-keratin autoantibodies; DLCO/VA, decrease in carbon monoxide diffusion capacity divided by alveolar volume; FCV, forced vital capacity; $\mathrm{HAQ}$, Health Assessment Questionnaire.

Statistical analysis was undertaken using $\chi^{2}$ tests, nonparametric tests, and multiple logistic regression.

\section{RESULTS}

\section{Study population}

We included 120 SSc patients (102 (85\%) of whom were women) with a median (range) age of 56.5 (20 to 90) years and a disease duration of 6 ( 0 to 42 ) years; 68 patients $(57 \%)$ had limited cutaneous disease and 52 (43\%) had diffuse cutaneous disease. Detailed characteristics of the SSc patients are provided in table 1 .

We included 44 control subjects:

- 22 women with rheumatoid arthritis with a median (range) age of 62.5 (38 to 75 ) years and a disease duration of 17 ( 3 to 39 ) years; $20(91 \%)$ of these patients tested positive for RF;

- 20 patients who had had digital trauma (eight men, 12 women) with a median (range) age of 52 (22 to 71) years.

\section{Results of radiography}

Twenty two SSc patients (18\%) had both erosion and joint space narrowing, which were considered to define arthritis for the purpose of this study. Radiological demineralisation was detected in 28 patients $(23 \%)$, and $26(22 \%)$ had distal phalange resorption. Flexion contracture was observed in 32 patients $(27 \%)$ and extra-articular calcifications in $28(23 \%)$. The radiological findings for patients with SSc are detailed in table 2. The more prevalent radiological patterns of SSc patients are illustrated in fig 1, panels A to C.

Twenty patients (90\%) with rheumatoid arthritis had erosive changes involving mainly the wrists (95\%) and metacarpophalangeal (MCP) joints (75\%). One patient with digital trauma had distal interphalangeal joint space narrowing and another had radiological demineralisation. The interobserver variation coefficient was 0.08 .

\section{ASSOCIATIONS BETWEEN RADIOLOGICAL FINDINGS AND SSC FEATURES \\ Joint pattern}

We found no significant difference between patients with and without arthritis $(n=22 / 120)$ or erosion $(n=25 / 120)$ in terms of disease duration $(p=0.39)$, cutaneous subtype $(p=0.64)$, occurrence of terminal tuft resorption $(p=0.06)$, or presence of RF $(p=0.85)$ and anti-CCP2 antibodies $(p=0.34)$. All SSc patients with erosive disease tested negative for AKA. An overlapping form with rheumatoid arthritis (symmetrical erosions) was seen in only two cases, both of whom tested positive for RF and anti-CCP antibodies. High C reactive protein concentrations ( $>10 \mathrm{mg} / \mathrm{l} ; \mathrm{n}=2 \mathrm{l}$ / $120)$ and an HAQ disability score $>1.5(n=29 / 120)$ were associated with the presence of arthritis (9/21 with increased $C$ reactive protein $v 13 / 99$ without increased $C$ reactive protein, $\mathrm{p}=0.004$; and 11/29 with high HAQ $v 11 / 91$ without high HAQ, $p=0.004)$. In multiple logistic regression analysis modelling increased $C$ reactive protein value $(>10 \mathrm{mg} / \mathrm{l})$, increased HAQ $(>1.5)$, cutaneous subtype, and disease duration longer than five years, we found that an increased $C$ reactive protein value $(p=0.03)$ and increased HAQ $(p=0.02)$ were associated with arthritis as the dependent variable.

\section{Bone pattern}

The prevalence of radiological demineralisation was higher in subjects with arthritis (12/22 $v 16 / 98$ for patients without arthritis; $\mathrm{p}=0.0001$ ) and high $\mathrm{C}$ reactive protein concentrations (9/21 $v 19 / 99$ for patients without increased $C$ reactive protein; $\mathrm{p}=0.04$ ).

Acro-osteolysis was found to be significantly associated with digital ulcers (16/37 $v$ 10/83 for patients without digital ulcers; $\mathrm{p}=0.0001$ ), intravenous prostacyclin requirement $(10 / 20 \vee 16 / 100$ for untreated patients; $p=0.005)$, extraarticular calcification (13/28 $v 13 / 92$ for patients without calcinosis; $\mathrm{p}=0.001$ ), and pulmonary arterial hypertension $(8 / 17 v 18 / 103$ for patients without PAH; $p=0.015)$. Digital
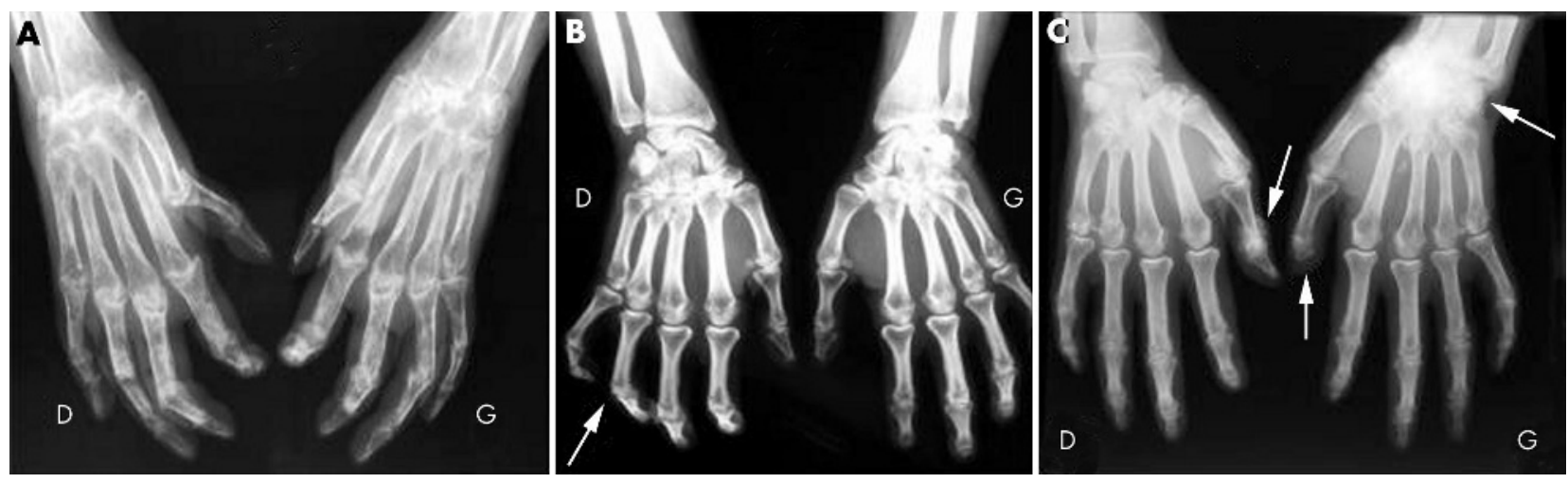

Figure 1 Main hand radiological pattern of patients with systemic sclerosis: erosion and arthritis (A); flexion contracture (B); tumoral calcinosis of the wrist with concomitant acro-osteolysis involving a middle phalange of the thumb and calcinosis (C). 


\begin{tabular}{ll}
$\begin{array}{l}\text { Table } 2 \\
\text { sclerosis }\end{array}$ & Radiological findings in patients with systemic \\
\hline & SSc patients \\
Radiological findings & ( $=120)$ \\
\hline Joint pattern & $25(21 \%)$ \\
Erosion & $17 / 25(68 \%)$ \\
Wrists & $9 / 25(36 \%)$ \\
MCP & $10 / 25(40 \%)$ \\
PIP & $18 / 25(72 \%)$ \\
DIP & $35(28.5 \%)$ \\
Joint space narrowing & $13 / 35(37.1 \%)$ \\
Wrists & $12 / 35(34.3 \%)$ \\
MCP & $14 / 35(40 \%)$ \\
PIP & $25 / 35(71.4 \%)$ \\
DIP & $22(18 \%)$ \\
Arthritis (erosion+joint space narrowing) & \\
Bone pattern & $28(23 \%)$ \\
Radiological demineralisation & $28(23 \%)$ \\
Bone resorption & $26(22 \%)$ \\
Acro-osteolysis & $2(2 \%)$ \\
Resorption of distal ulna & \\
Soft tissue pattern & $28(23 \%)$ \\
Calcinosis & $32(27 \%)$ \\
Flexion contracture & \\
\hline Values are $n$ (\%). & \\
DIP, distal interphalangeal joint; MCP, metacarpophalangeal joint; PIP, \\
proximal interphalangeal joint.
\end{tabular}

tuft resorption was not associated with the presence of antitopoisomerase I antibodies $(p=0.054)$. In multiple logistic regression analysis taking into account digital ulcers, prostacyclin use, calcinosis, and $\mathrm{PAH}$ as independent variables, we found that digital ulcers $(p=0.004)$, calcinosis $(\mathrm{p}=0.008)$, and PAH $(\mathrm{p}=0.03)$ were associated with acroosteolysis as the dependent variable.

\section{Soft tissue pattern}

Flexion contracture was associated with the diffuse cutaneous form $(24 / 52 \vee 8 / 68$ for patients with the limited cutaneous subtype; $\mathrm{p}<0.0001$ ), HAQ disability scores $>1.5$ $(13 / 29 v 19 / 91$ for patients without high HAQ score; $p=0.02)$, and pulmonary fibrosis $(21 / 58 \vee 11 / 62$ for patients without pulmonary fibrosis; $\mathrm{p}=0.04$ ). No relation was found between fibrosis severity as estimated from forced vital capacity $(p=0.33)$, positivity for anti-topoisomerase I antibodies $(\mathrm{p}=0.20)$ and flexion contracture in univariate analysis. In multiple logistic regression analysis taking into account cutaneous subtype, pulmonary fibrosis on CT, decreased forced vital capacity below 75\% of predicted value, and increased HAQ score $(>1.5)$, we found that the diffuse cutaneous form $(p<0.015)$ and high HAQ score $(p=0.007)$ were associated with flexion contraction as the dependent variable.

Calcinosis tended to be associated with erosion (10/25 v 18/ 95 in patients without erosion; $\mathrm{p}=0.05)$ and was associated with digital ulcers (15/37 $v 13 / 83$ in patients without digital ulcers; $p=0.006$ ). The frequency of calcinosis was similar in patients with diffuse and limited cutaneous forms and was not associated with the presence of anti-centromere antibodies $(p=0.8)$. In multiple logistic regression analysis taking into account erosion and arthritis on $x$ ray, digital ulcers, cutaneous subtype, and positivity for anti-centromere antibodies, we found that digital ulcers $(p=0.002)$ were associated with calcinosis as the dependent variable.

In all patients with calcinosis, lesions were preferentially located on the dominant hand, which was involved in all cases, with eight of 28 patients having calcinosis of the dominant hand only and 20 of 28 having lesions on both hands, with the lesions on the dominant hand being more severe.

\section{DISCUSSION}

Our results highlight the striking level of hand involvement in SSc, as evaluated by systematic $x$ ray. The main features of this involvement were as follows: erosion $(21 \%)$, joint space narrowing $(28 \%)$, concomitant erosion and joint space narrowing (18\%), radiological demineralisation (23\%), acroosteolysis $(22 \%)$, flexion contracture $(27 \%)$, and calcinosis $(23 \%)$. Our data show that flexion contracture occurs in the diffuse cutaneous SSc subtype and that acro-osteolysis and calcinosis occur in patients with the more severe vascular complications.

The prevalence of articular erosion in SSc has been estimated at between $5 \%$ and $40 \%$ in previous studies ${ }^{1-8}$ and was $21 \%$ in our series. Only two SSc patients with erosions fulfilled the ACR criteria for classical rheumatoid arthritis, consistent with the existence of primary erosive arthropathy in SSc unexplained by overlap with rheumatoid arthritis, as suggested by La Montagna et al. ${ }^{3}$ Thirty five patients $(28 \%)$ had joint space narrowing, mostly involving the DIP joints $(71 \%)$, consistent with published data. ${ }^{12}{ }^{24} \mathrm{We}$ found that 18 of 25 patients with erosions had erosive changes in the DIP joints compatible with erosive osteoarthritis. Most of our patients were post-menopausal women, and were therefore prone to erosive osteoarthritis. We could not rule out the possibility of such an arthropathy, unrelated to SSc, occurring in our patients. Arthritis was associated with high $\mathrm{C}$ reactive protein concentration $(>10 \mathrm{mg} / \mathrm{l})$, suggesting that inflammatory articular involvement may partly account for the unusual inflammatory syndrome observed in SSc patients. The treatment of arthritis in SSc patients has not yet been standardised. By analogy with rheumatoid arthritis, methotrexate and low doses of corticosteroids are usually given, but these alternative treatments have not been evaluated. Patients with rheumatoid arthritis in our series had severe erosive changes which is consistent with their recruitment in a tertiary hospital unit.

Radiological demineralisation was observed in $23 \%$ of previously reported SSc patients. ${ }^{1325}$ Our data suggest that radiological demineralisation is associated with arthritis and systemic inflammation. Acro-osteolysis is one of the most characteristic radiographic findings in SSc, in which terminal digital tuft resorption tends to occur. ${ }^{1-14}$ Bone resorption generally begins on the tuft, particularly on the palmar surface of the bone. Continued resorption leads to "pencilling" or sharpening of the phalanx and, in severe cases, much or all of the distal phalange may be destroyed, resulting in the tapering of fingers. In rare instances, the middle phalanges may also be involved (fig lC). Bony erosion of the phalanges in the hand has been reported to occur in $29-80 \%$ of SSc patients. $^{13101314}$ These very disparate results may be accounted for by differences in the number of patients included in these studies (from 21 to 100 patients) and the combination of retrospective and prospective data. In our prospective evaluation, we identified 26 patients (22\%) with acro-osteolysis and a significant association of acro-osteolysis with vascular digital and systemic complications in univariate and multivariate analysis. Although any pathogenic link emerging from an observational study should be interpreted with caution, our results are consistent with vascular injury playing a role in such lesions, possibly because of repeated vasospasm, as suggested by Sharer and Smith. ${ }^{26}$ Digital ulcerations were more severe in the acro-osteolysis group than in the other groups, with this group having a significant requirement for intravenous prostacyclin analogues, suggesting that acro-osteolysis may occur in the patients with the most severe vascular complications. Anti-topoisomerase 1 
antibodies were detected more often in patients with acroosteolysis than in other patients, but the difference was not significant. Conversely, Takahashi et al reported six patients with acro-osteolysis and anti-centromere antibodies, but no anti-topoisomerase 1 antibodies. ${ }^{27}$

Flexion contracture results from fibrotic changes in the skin-the hallmark of SSc and a source of functional disability. ${ }^{16}$ We found that flexion contracture was significantly associated with the diffuse cutaneous form and a high HAQ score in univariate and multivariate analysis. These associations highlight the greater impairment of hand function in the diffuse subgroup, as reported by Brower and Poole. ${ }^{16}$ La Montagna et al also found a significant association between a radiological fibrotic pattern, including digital flexion contracture, and a severity score for skin involvement. ${ }^{328}$ These results suggest that physical therapy for these patients should be evaluated.

Amorphous calcification, particularly of the hand, appears to be common in patients with $\mathrm{SSc}^{1211}{ }^{12}$; localisation to this site has been recorded in $73-86 \%$ of patients with calcinosis. ${ }^{28}$ Digital calcifications were seen in $10-50 \%$ of SSc patients in previous studies $^{12-11}$ and in $23 \%$ in our series. Calcinosis was not restricted to patients with the limited form of the disease. $^{29}$ This result is consistent with previous studies showing that the occurrence of calcinosis does not depend on cutaneous subtype. ${ }^{3031}$ Nishikai et al and Vayssairat et al suggested an association between anti-centromere antibodies and the presence of subcutaneous calcification ${ }^{29}{ }^{32}$; our data do not support this finding. One of our major findings was the association in univariate and multivariate analysis between calcinosis and vascular complications, patients with calcinosis being more likely to have a history of digital ulcers. Vayssairat et al suggested that capillary injury involvement was greater in patients with subcutaneous calcinosis. ${ }^{29}$ Vascular injury and ischaemia may therefore be involved in the pathogenesis of such lesions, suggesting that vasodilators such as calcium channel blockers could be used in treatment. ${ }^{33-35}$ Nevertheless, evidence that diltiazem has a positive effect on calcinosis remains to be confirmed ${ }^{29}$ and further investigations are warranted.

Calcification often occurs at sites of chronic stress, ${ }^{12}$ such as the radial sides of the fingers apposing the thumb and the soft tissues around bony prominences, suggesting a role of trauma in the pathogenesis of these deposits. ${ }^{36}$ This role is highlighted by the observation in our series and published studies of more severe calcification in the dominant hand ${ }^{11}$ and by the absence of calcinosis (or bone resorption) in fingers protected from the stress of normal activity because of previous injury. ${ }^{26}$

Calcinosis was more frequent in patients with erosive lesions. Some studies have suggested that calcinosis is involved in the development of destructive arthritis owing to the massive deposition of calcium apatite crystals in joints subject to phagocytosis by synovial fluid cells. ${ }^{37-39}$ However, the pathogenic role of apatite crystals in arthritis remains unclear.

Magnetic resonance imaging may facilitate future studies of hand involvement in SSc, making it possible to determine the involvement of various tissues. ${ }^{3}$

\section{Conclusion}

Arthritis, erosions, joint space narrowing, radiological demineralisation, acro-osteolysis, flexion contracture, and calcinosis were the main features of radiological hand involvement in this series of patients with SSc. These findings confirm that hand involvement is common and leads to disability. Flexion contracture was a source of disability and occurred in patients with the diffuse cutaneous form of the disease, consistent with the fibrosis and functional impairment observed in this form. Calcinosis and acro-osteolysis were both associated with vascular digital and systemic complications, highlighting the role of vascular injury in such lesions.

\section{Authors' affiliations}

J Avouac, J Wipff, N Assous, A Kahan, Y Allanore, Department of Rheumatology A, René Descartes University, School of Medicine, Cochin Hospital, Paris, France

H Guerini, A Chevrot, Department of Radiology B, René Descartes

University, School of Medicine, Cochin Hospital

\section{REFERENCES}

1 Baron M, Lee P, Keystone EC. The articular manifestations of progressive systemic sclerosis (scleroderma). Ann Rheum Dis 1982;41:147-52.

2 Tuffanelli DL, Winkelmann RK. Systemic scleroderma. A clinical study of 727 cases. Arch Dermatol 1961;84:359-71.

3 La Montagna G, Sodano A, Capurro V, Malesci D, Valentini G. The arthropathy of systemic sclerosis: a 12 month prospective clinical and imaging study. Skeletal Radiol 2005;34:35-41.

4 La Montagna G, Baruffo A, Tirri R, Buono G, Valentini G. Foot involvement in systemic sclerosis: a longitudinal study of 100 patients. Semin Arthritis Rheum 2002;31:248-55.

5 Brun B, Serup J, Hagdrup H. Radiological changes of the hands in systemic sclerosis. Acta Derm Venereol 1983;63:349-52.

6 Lovell CR, Jayson MI. Joint involvement in systemic sclerosis. Scand J Rheumatol 1979;8:154-60.

7 Bassett LW, Blocka KL, Furst DE, Clements PJ, Gold RH. Skeletal findings in progressive systemic sclerosis (scleroderma). Am J Roentgenol 1981;136:1121-6.

8 Blocka KL, Bassett LW, Furst DE, Clements PJ, Paulus HE. The arthropathy of advanced progressive systemic sclerosis. A radiographic survey. Arthritis Rheum 1981;24:874-84.

9 Arnaud M, Treves R, Desproges-Gotteron R, Arnaud JP, Charissoux JL, Pecout C. Unusual calcifications in scleroderma. Apropos of a case. Rev Rhum Mal Osteoartic 1988;55:721-2.

10 Kempt-Harper RA, Jackson DC. Progressive systemic sclerosis. Br J Radiol 1967;26:847.

11 Schlenker JD, Clark DD, Weckesser EC. Calcinosis circumscripta of the hand in scleroderma. Bone Joint Surg Am 1973;55:1051-6.

12 Resnick D. Scleroderma (progressive systemic sclerosis). Diagnosis of bone and joint disorders, 2nd edition. Philadelphia: WB Saunders, 1988:1191-216.

13 Yune HY, Vix VA, Klatte EC. Early fingertip changes in scleroderma. JAMA 1971;215:1113-16.

14 Poznanski AK. The hand in radiologic diagnosis. Philadelphia: WB Saunders, 1974.

15 Mau W, Listing J, Huscher D, Zeidler H, Zink A. Employment across chronic inflammatory rheumatic diseases and comparison with the general population. J Rheumatol 2005;32:721-8.

16 Brower LM, Poole JL. Reliability and validity of the Duruoz Hand Index in persons with systemic sclerosis (scleroderma). Arthritis Rheum 2004:51:805-9.

17 Poole JL, Steen VD. The use of the Health Assessment Questionnaire (HAQ) to determine physical disability in systemic sclerosis. Arthritis Care Res $1991 ; 4: 27-31$

18 Sandqvist G, Eklund M. Hand Mobility in Scleroderma (HAMIS) test: the reliability of a novel hand function test. Arthritis Care Res 2000;13:369-74.

19 Backman C, Mackie H, Harris J. Arthritis hand function test: development of a standard assessment tool. Occup Ther J Res 1991;11:245-56.

20 Poole JL, Gallegos M, O'Linc S. Reliability and validity of the Arthritis Hand Function Test in adults with systemic sclerosis (scleroderma). Arthritis Care Res 2000;13:69-73.

21 Leroy EC, Black C, Fleischmajer R, Jablonska S, Krieg T, Medsger TA, et al. Scleroderma (systemic sclerosis): classification, subset and pathogenesis. $J$ Rheumatol 1988;15:202-5.

22 Valentini G, Medsger TA, Silman AJ, Bombardieri S. Conclusion and identification of the core set of variables to be used in clinical investigations. Clin Exp Rheumatol 2003;21:S47-8.

23 Allanore Y, Borderie D, Meune C, Cabanes L, Weber S, Ekindjian OG, et al. $\mathrm{N}$-terminal pro-brain natriuretic peptide as a diagnostic marker of early pulmonary artery hypertension in patients with systemic sclerosis and effects of calcium-channel blockers. Arthritis Rheum 2003;48:3503-8.

24 Brower AC, Resnick D, Karlin C. Unusual articular changes of the hand in scleroderma. Skel Radiol 1979:4:119-23.

25 Blocka KL, Bassett LW, Furst DE, Clements PJ, Paulus HE. The arthropathy of advanced progressive systemic sclerosis. A radiographic survey. Arthritis Rheum 1981;24:874-84.

26 Scharer L, Smith DW. Resorption of the terminal phalanges in scleroderma. Arthritis Rheum 1969;12:51-63.

27 Takahashi M, Okada J, Kondo $\mathrm{H}$. Six cases positive for anti-centromere antibodies with ulcer and gangrene in the extremities. Br J Rheumatol 1997;36:889-93.

28 Medsger TA, Bombardieri S, Czirjak L, Scorza R, Della Rossa A, Bencivelli W. Assessment of disease severity and prognosis. Clin Exp Rheumatol 2003;21:S42-6. 
29 Vayssairat M, Hidouche D, Abdoucheli-Baudot N, Gaitz JP. Clinical significance of subcutaneous calcinosis in patients with systemic sclerosis. Does diltiazem induce its regression? Ann Rheum Dis 1998;57:252-4.

30 Wollheim FA. Classification of systemic sclerosis. Visions and reality. Rheumatology (Oxford) 2005;44:1212-16.

31 Akesson A, Wollheim FA. Organ manifestations in 100 patients with progressive systemic sclerosis: a comparison between the CREST syndrome and diffuse scleroderma. Br J Rheumatol 1989;28:281-6.

32 Nishikai $M$, Itoh K, Sato A. Calcinosis and the anticentromere antibody: its clinical, radiological and immunogenetic aspects. $\mathrm{Br} J$ Rheumatol 1992;31:9-12.

33 Farah MJ, Palmieri GM, Sebes Jl, Cremer MA, Massie JD, Pinals RS. The effect of diltiazem on calcinosis in a patient with the CREST syndrome. Arthritis Rheum 1990;33:1287-93.
34 Dolan AL, Kassimos D, Gibson T, Kingsley GH. Diltiazem induces remission of calcinosis in scleroderma. Br J Rheumatol 1995;34:576-8.

35 Palmieri GM, Sebes Jl, Aelion JA, Moinuddin M, Ray MW, Wood GC, et al. Treatment of calcinosis with diltiazem. Arthritis Rheum 1995;38:1646-54.

36 Rodnan GP, Medsger TA. The rheumatic manifestations of progressive systemic sclerosis (scleroderma). Clin Orthop Relat Res 1968;57:81-93.

37 Brandt KD, Krey PR. Chalky joint effusion. The result of massive synovial deposition of calcium apatite in progressive systemic sclerosis. Arthritis Rheum 1977:20:792-6

38 Resnick D, Scavulli JF, Goergen TG, Genant HK, Niwayama G. Intra-articular calcification in scleroderma. Radiology 1977; 124:685-8.

39 Reginato AJ, Schumacher HR. Synovial calcification in a patient with collagen-vascular disease: light and electron microscopic studies. J Rheumatol 1977;4:261-71.

\section{Get published within days of acceptance with ARD}

We are delighted to announce that the Annals of the Rheumatic Diseases launched a "publish ahead of print" programme in February 2004. Selected papers are fast tracked and published online months before they appear in the print journal.

Papers of major significance to the international rheumatology community are published within days of acceptance. The first published article is the raw accepted manuscript; edited and typeset versions are also published as soon as they are available.

In addition to being available on ARD Online, the publish ahead of print articles are searchable through PubMed/ Medline-establishing primacy for your work. They are linked from the ARD Online home page.

To take advantage of this "publish ahead of print" programme submit your papers to the Annals of the Rheumatic Diseases using our online submission and review system Bench >Press (http://submit-ard.bmijournals. com). For further information contact ARD@bmigroup.com. 